\title{
A Trip Down the Bronchus- A Tracheostomy Complication in COVID Pandemic
}

\author{
Sauradeep Das $^{1} \cdot$ Saranya Thangavel $^{1} \cdot$ Kalaiarasi Raja $^{1}\left[\right.$ Tunil Kumar Saxena $^{1}$
}

Received: 1 July 2021/Accepted: 8 August 2021/Published online: 22 August 2021

(C) Association of Otolaryngologists of India 2021

\begin{abstract}
Foreign body of the trachea and the bronchus are critical emergencies which can lead to life threatening complications. But the advent of the 2019 novel corona virus disease, pandemic has dramatically changed the comfort of these procedures since aerosol generating medical procedures pose a risk and spread of infection to the health care workers. Even the patients are uncomfortable visiting the hospital due to the fear of acquiring the COVID infection.A 41-year-old obese female with grade 4 subglottic stenosis status post tracheostomy presented with foreign body Fuller's tracheostomy tube flange in the right bronchus during the COVID 19 pandemic. The patient had delayed presentation to the hospital due to fear of getting exposed to COVID and poor access to health care facilities due to lockdown imposed in various places in the nation. The patient was tested for COVID and taken up for surgery where rigid bronchoscopy and foreign body removal was done via the tracheostoma. The details of the procedure, challenges faced during the procedure, the effect of the COVID pandemic on the patients and hospital staffs are discussed. The complications of the broken tracheostomy tube can be most efficiently dispelled by proper tube care by the attenders and frequent tube change.
\end{abstract}

Keywords Rigid bronchoscopy · Trachoestomy · COVID-19 · Fullers tube

Kalaiarasi Raja

kalaiarasi004@gmail.com

1 Department of ENT, Jawaharlal Institute of Post Graduate Medical Education and Research (JIPMER), Pondicherry 605006, India

\section{Introduction}

Foreign body $(\mathrm{FB})$ of the trachea and the bronchus are critical emergencies which can lead to life threatening complications. Generally rigid bronchoscopy with optical forceps for optical imaging and surgical removal is done. But the advent of the 2019 novel corona virus disease (COVID 19), pandemic has dramatically changed the comfort of these procedures since Aerosol Generating Medical Procedures (AGMP) pose a risk and spread of infection to the health care workers especially so among the Otorhinolaryngologists $[1,2]$. Even the patients are uncomfortable visiting the hospital due to the fear of acquiring the COVID infection. This led to a delay in the diagnosis and the treatment for the patients leading to a more difficult bronchoscopy and more exposure to aerosols for the healthcare workers.

\section{Case Report}

A 41-year-old obese female with history of suicidal hanging and prolonged intubation, for which she was tracheostomised three years before, had come with complaints of discomfort in the neck and difficulty in speaking with the Fullers tube for the past seven days. She was on treatment for bipolar disorder for the last ten years. She had no known contact with any COVID patients, travel, or any other risk factors for COVID-19. The patient had no other symptoms of COVID-19 disease at the time of admission (no fever, sore throat, rhinorrhoea) but had paroxysmal cough and mild difficulty in breathing. The patient had no stridor. On examination, it was found that the Fullers tube that she was using had its right flange missing. Plain X-ray Chest and Soft tissue neck lateral views were taken which 
showed the Fullers tracheostomy tube flange in the right bronchus of the patient (Fig. 1). Due to the COVID pandemic and her psychiatric illness, her family members had ignored her complaints and asked her to continue the psychiatric medications at home.

The patient was worked up and planned for an elective rigid bronchoscopy and foreign body removal under general anaesthesia as the patient was stable. A COVID Real Time Polymerase Chain Reaction swab test was negative. As she had a grade 4 subglottic stenosis, bronchoscopy could not be done trans orally. So, FB removal via the tracheostoma was planned. The patient was in supine position with neck extended with sandbag under the shoulder. Karl Storz Rigid bronchoscope size 5 was introduced through the tracheostoma and the appropriate optical forceps with Hopkins's rod endoscope was used to retrieve the FB. The patient was ventilated via the bronchoscope. The foreign body (Fuller's tube flange) was visualised with granulation tissue around the foreign body in the right bronchus and in carina (Fig. 2). Bleeding was noted from the granulation tissue which was stopped using lin 2 lakhs adrenaline soaked pattey. The Fuller's tube flange was held using the serrated forceps, but the flange could not be retrieved through tracheostoma as stoma was contracted in size after the breakage of the tube. The tracheostoma was revised after $2 \%$ lignocaine with adrenaline infiltration. A cruciate incision was given around the stoma which had contracted in seven days duration. The flange was removed in 2 pieces. A follow up X-ray was done for the patient and was found to be normal. The patient was discharged and was fine on follow up after 3 months.

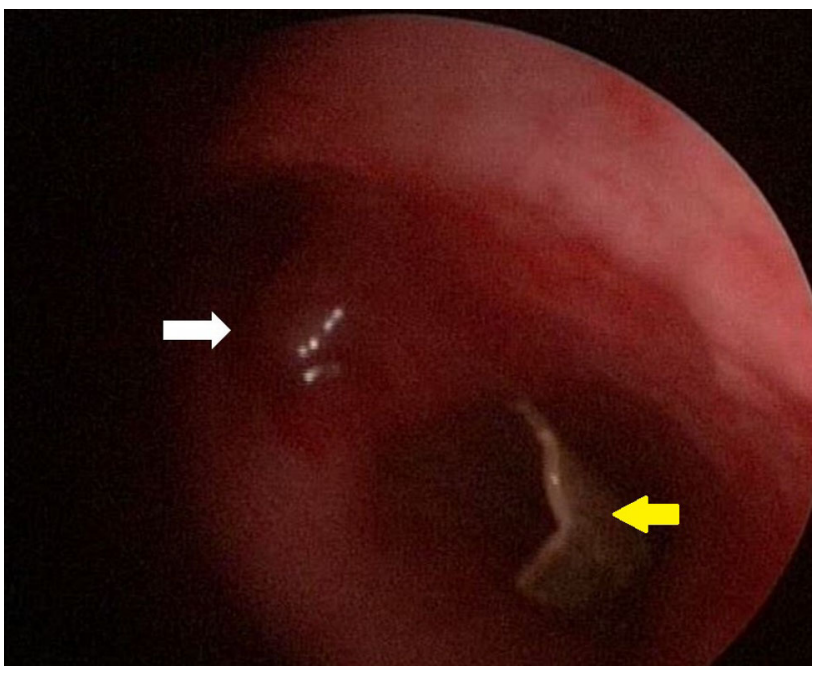

Fig. 2 Telescopic view of the foreign body-broken fuller tracheostomy tube flange (yellow arrow) in the right main bronchus with granulation tissue in the carina (white arrow)

\section{Discussion}

A broken tracheostomy tube is an unusual foreign body of the bronchus. It is one of the rare complications of tracheostomy. The first case of such type was reported by Bassoc and Boe in 1960 [3]. The prospect of dealing with such an unusual foreign body like a broken flange of the tracheostomy tube in the tracheobronchial tree can be deceptive and a challenge even for a skilful surgeon.

Foreign body aspiration can present as a life-threatening emergency with acute airway obstruction and respiratory failure which require urgent intervention. Most patients present acutely and only very few presents with an indolent chronic symptom. Cough is the most common symptom and is present in two-thirds of the patients. Other
Fig. 1 Plain $X$ ray chest Antero posterior(AP) view (A) and soft tissue neck lateral (B) view showing radio opaque broken flange of the Fuller s tracheostomy tube (white arrow) in right main bronchus

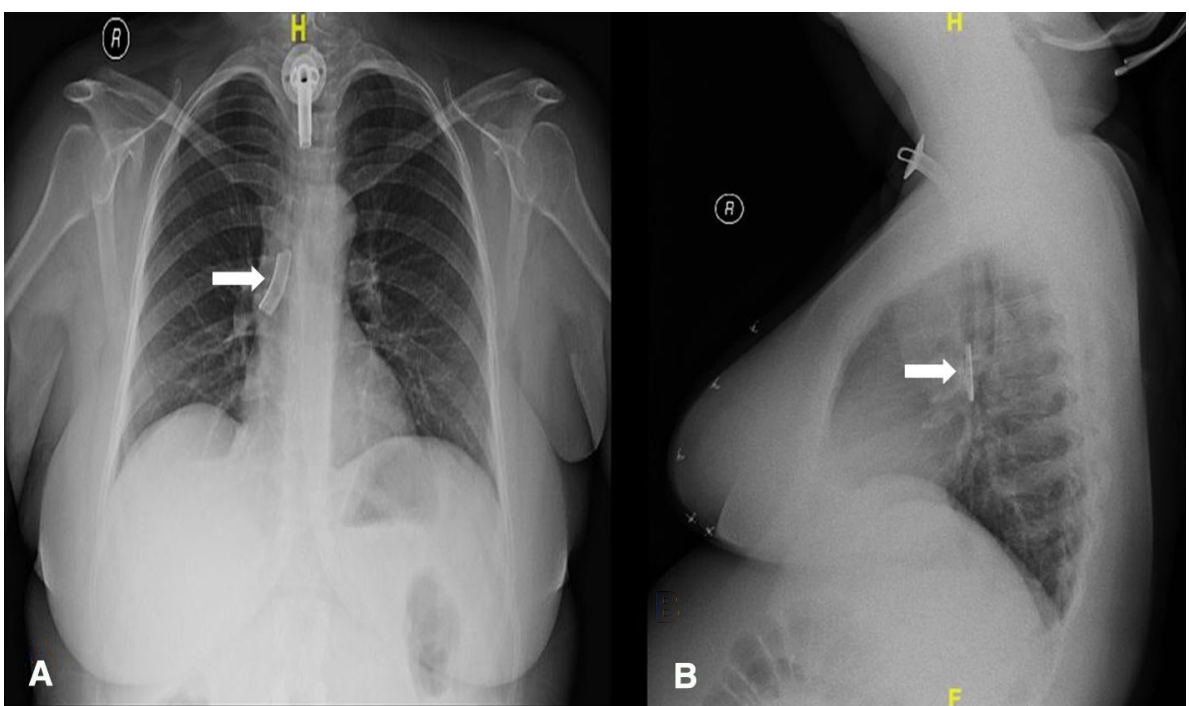


commonly reported symptoms include haemoptysis, fever, and dyspnoea [4]. The patient presented with chronic cough with breathing difficulty which was considered trivial by her care takers. The reasons for delayed presentation to the hospital are absence of acute symptoms like choking, fear of COVID pandemic, and poor medical access.

Intraoperative challenges were positioning the patient for bronchoscopy, the preferred position is Boyle's position. But when removal was planned through tracheostoma, neck extension is preferred which was achieved with sandbag under the shoulder. Since patient was obese with short neck, positioning the patient was a challenge.

Delayed presentation to the hospital resulted in stomal stenosis and granulation tissue around the broken flange resulting in difficult retrieval of the FB. A cruciate incision was given in the stoma to enlarge the stoma to facilitate the removal of broken flange through the stoma. Adrenaline soaked cottonoids were used to control the bleeding from the granulation tissue and for better visualization of the FB. Because of delayed presentation, the broken flange could not be removed in single piece but removed in two pieces as the broken piece was in alkaline bronchial secretion. Because of these challenges, the procedure lasted for 20 min.

The metallic tracheostomy tubes have been recommended to be made from $45 \%$ silver, $15 \%$ copper, $24 \%$ cadmium and $16 \%$ zinc [5]. It has been analysed and found that the zinc component of the tracheostomy tube is more responsible for the corrosion. Indian metallic tubes have a very thin layer of silver plating which wears out in two to three weeks [6]. Most of the cases of the broken tubes occur due to poor tracheostomy care and the failure of the patients to do a regular tube care and tube change. This allowed the alkaline secretions and the moisture from the tracheobronchial tree to accumulate over the tube. This reacts with the metallic tube and leads to corrosion known as "season cracking " [7]. The break of the flanges might be attributed to the defective manufacture or braising of the metal tubes leading to detachment of the tube from the neck plate [8]. In some cases, remoulding of the flanges repeatedly to fit the inner cannula snugly or to facilitate insertion through a narrowed stoma (as in our patient) can lead to weakening of the site of attachment of the flanges.

A proper tracheostomy tube care is an essential part of preventing the occurrence of such instances, which includes proper tube care, regular tube changes and frequent suctioning using proper suction equipment. Keeping the stoma clean with regular dressings and clearing the secretions prevent granulation tissue and further stomal infection which might later lead to the contracture of the stoma $[9,10]$.
Time factor is another essential factor responsible for the fracture of the flange of tracheostomy tube. This is especially true at the time of this COVID-19 pandemic which has resulted in a fear and apprehension among patients to visit the hospital. This was true in our patient who had not come to the hospital for one year duration.

Furthermore, as in our case, the patients have an apprehension to come to the hospital because of COVID pandemic. This leads to a delay in the diagnosis and treatment of the patients. This delay leads to an increased edema and granulation tissue formation around the foreign body as in the patient and thus further complicated the procedure.

Bronchoscopy being an AGMP must be done with the least time using a short GA and decreased complications to the patient as well as to decrease the exposure of the health care workers to the aerosols during the procedure. Occurrence of the granulation tissue and edema in the bronchus led to a prolongation of the procedure and an increased risk for both patients and healthcare workers.

In patients with subglottic stenosis with FB in the trachea and bronchus, bronchoscopy via the tracheostoma has been a feasible approach for the removal of the foreign body [11]. But due to the COVID-19 pandemic, precautions must be taken like wearing personal protective equipment including N-95 masks, face shield, protective gown, and gloves despite a negative COVID RT PCR report, the patient with COVID 19 may be asymptomatic or presymptomatic [12].

\section{Conclusion}

The complications of the broken tracheostomy tube can be most efficiently dispelled by proper tube care by the attenders and frequent tube change. In case of a foreign body bronchus, an early and prompt action is mandatory especially during these times of COVID 19 pandemic. Proper planning with necessary precautions will help in a safe and early removal of such foreign bodies without any hazards to health workers and decreased timing of the procedure and hence also decreased aerosol production.

Funding The authors did not receive support from any organization for the submitted work.

\section{Declarations}

Conflicts of interest The author declares no conflict of interest.

Informed Consent Obtained from the patient for participation. 


\section{References}

1. Tran K, Cimon K, Severn M, Pessoa-Silva CL, Conly J (2012) Aerosol generating procedures and risk of transmission of acute respiratory infections to healthcare workers: a systematic review. PloS one. 7(4):e35797

2. Patel ZM, Fernandez-Miranda J, Hwang PH, Nayak JV, Dodd R, Sajjadi H, Jackler RK (2020) Precautions for endoscopic transnasal skull base surgery during the COVID-19 pandemic. Neurosurg 87(1):E66-E67

3. Hh B (1960) Broken tracheotomy tube as a foreign body. Lancet (London, England) 1(7132):1006-1007

4. Chen CH, Lai CL, Tsai TT, Lee YC, Perng RP (1997) Foreign body aspiration into the lower airway in Chinese adults. Chest 112(1):129-133

5. Parida PK, Raja Kalaiarasi AA, Saxena SK (2020) Factors Associated with Fracture and Migration of Tracheostomy Tube into Trachea in Children: A Case Series. Iranian J Otorhinolaryngol 32(113):379

6. Chaturvedi VN, Gupta NB (1995) Study of size and manufacturing defects of various tracheostomy tubes. Ind J Otolaryngol Head Neck Surg 47(3):221-225
7. Bhargava SK, Bhat N, Bhargava KB (1993) Broken tracheostomy introducer-an unusual tracheobronchial foreign body. J Laryngol Otol 107(5):463-464

8. Poorey VK, Iyer A (2001) Unusual foreign body (broken tracheostomy tube) in left main bronchus. Ind J Otolaryngol Head Neck Surg 53(3):233-234

9. Oberwaldner B, Eber E (2006) Tracheostomy care in the home. Paediatr Respir Rev 7(3):185-190

10 Reddy RZ, Carter YM, Hsia DW (2018) Successful removal of a chronic aspirated foreign body after twelve years. Case reports pulmonol. https://doi.org/10.1155/2018/8241591

11. Fraga JC, Pires AF, Komlos M, Takamatu EE, Camargo LG, Contelli FH (2003) Bronchoscopic removal of foreign body from airway through tracheotomy or tracheostomy. Jornal de pediatria 79(4):369-372

12. Krall J, Ali M, Maslonka M, Pickens A, Bellinger C (2020) Bronchoscopy in the COVID-19 Era. Clinical Pulmonary Med 27(6):198

Publisher's Note Springer Nature remains neutral with regard to jurisdictional claims in published maps and institutional affiliations. 